\title{
Sarcomatoid carcinoma of the urinary bladder: A case report
}

\author{
A U Mallik ${ }^{1}$, M Z Rahman², M M R Sarker ${ }^{3}$
}

\begin{abstract}
Background: Sarcomatoid carcinoma of the urinary bladder is a very rare variety of transitional cell carcinoma (TCC) with a prominent component of spindle-shaped cell.

The incidence of this tumor is $0.5 \%$ of all bladder tumors.

Case report: Herein, we report a case of sarcomatoid carcinoma of urinary bladder of a farmer of 70 years, a smoker with the complaints of lower abdominal pain, mild burning during urination and 3 episodes of painless gross haematuria. USG of KUB revealed a cystic lesion in front of the anterior bladder wall. Partial cystectomy was carried out. Diagnosis was confirmed by histopathological study. Patient refused radiotherapy or chemotherapy and died after 7 months of surgery due to metastasis.
\end{abstract}

Key Words: TCC, Sarcomatoid carcinoma. Partial cystectomy

Introduction: Sarcomatoid carcinoma (SC) is an aggressive type of malignant tumor that begins in tissues which line or cover internal organs and frequently a poorly differentiated urothelial carcinoma that may be difficult to distinguish from a sarcoma.

$\mathrm{SC}$ is a very rare variety. The incidence rate of this tumor is $0.5 \%$ of all bladder tumors and has an aggressive behavior, yielding a poor prognosis despite different treatment modalities.

It has a variety of names such as sarcomatoid carcinoma, pseudo sarcoma, malignant mixed mesodermal / mullerian tumor, metaplastic carcinoma and spindle cell carcinoma. ${ }^{2}$ Herein, we report a case of sarcomatoid carcinoma of the urinary bladder that was treated by partial cystectomy and diagnosed with histopathological study. Since the guardian of the patient refused total cystectomy with chemotherapy and radiotherapy, the outcome was found to be poor.

Case report: A 70 year old male farmer with a habit of smoking came with the complaints of 3 episodes of painless gross hematuria within 1 and half months. He also complained of mild burning during urination and lower abdominal pain intermittent in nature. His past history and family history was unremarkable. On physical examination, no abnormality was detected. The blood chemistry was within normal limit except for mild anemia. Urine microscopic examination revealed pyuria. USG of abdomen revealed a cystic lesion in front of the anterior bladder wall the first time. In cystoscopy, a hemorrhagic area in the anterior bladder wall was found. Patient was discharged. After 1 month he again came to us with one episode of hematuria. This time, USG of lower abdomen was unremarkable. It was decided to explore the urinary bladder for the cystic lesion in front of the anterior bladder wall. A hard mass was found in the anterior bladder wall. A total cystectomy was planned, but patient's guardian refused, so partial cystectomy with removal of $1 \mathrm{~cm}$ bladder wall surrounding the mass was undergone. Specimen was sent for histopathological study.
Macroscopically $5 \times 4.5 \times 3 \mathrm{~cm}$ a tumor infiltrating the adipose tissue at the anterior wall of bladder was seen. Microscopically, this tumor infiltrated all layers of the bladder wall. Histopathological findings show a malignant tumor of sarcomatoid carcinoma. It is composed of malignant epithelial component with glandular differentiation in a background of areas having sarcoma like appearance. The features are consistent with area of sarcomatoid carcinoma

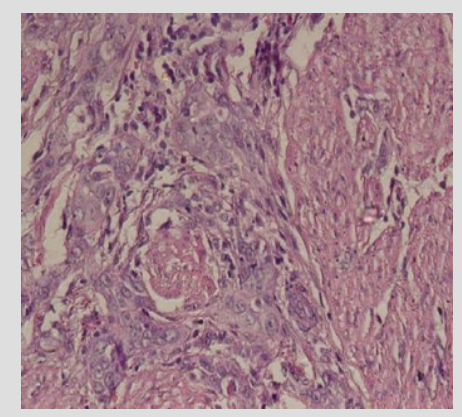

Figure 1: High power view of Sarcomatoid carcinoma. It is composed of malignant epithelial component with glandular differentiation and area having sarcoma like appearance. ( $\mathrm{H}$ and E x 200)

Patient passed away 7 months after surgery due to metastasis.

Discussion: SC is an aggressive form of locally advanced carcinoma and has high rates of recurrence. Biopsy for histopathological and immunohistochemical study is required for accurate diagnosis. The treatment options for SC will vary depending upon many factors having to do with the patient's over-all condition. ${ }^{3} \mathrm{SC}$ is composed predominantly of a sarcomatoid component and an obviously carcinomatous component. The sarcomatoid component is composed of a mixture of spindle cells, round cells, and pleomorphic giant cells. The carcinomatous component consisted of papillary or non-papillary high-grade TCC. In our case the sections of tissues shows a malignant epithelial component with glandular differentiation in a background of areas having sarcoma like appearance. The features are consistent with 


\section{Sarcomatoid carcinoma}

area of sarcomatoid carcinoma. ${ }^{3}$ One reported that of 4191 patients with bladder carcinoma, the incidence of SC was $0.3 \%{ }^{4}$ and $1.06 \%{ }^{5}$ in another report. As SC is a very rare tumor, data regarding etiology and outcome is also limited. Although, no definite risk factors for SC are identified to date, an association with radiation and cyclophosphamide therapy has been noted. The male female ratio is approximate $3: 14$. In general, SC presents a diagnostic difficulty if any definite abnormalities are not found in investigation, as was the case in our patient. ${ }^{5,6,7}$ We reported such a case of SC of urinary bladder treated with partial cystectomy. The median age at diagnosis is 60 to 70 years and most of the patients with SC of urinary bladder presents with painless hematuria as was in our case. Although the term sarcomatoid carcinoma and carcinosarcoma have been used together, the term sarcomatoid carcinoma has been used to describe malignant spindle cell type tumor with epithelial differentiation, while carcinosarcoma describes the tumor composed of both malignant epithelial and malignant soft tissue elements. ${ }^{8}$ In sarcomatoid carcinoma, the sarcomatous elements are chondrosarcoma, leiomyosarcoma, fibrosarcoma and rhabdomyosarcoma and epithelial elements consist of transitional cell carcinoma, small cell carcinoma, squamous carcinoma and other mixed carcinoma.

To differentiate sarcomatoid carcinoma and carcinosarcoma, immunohistochemical study is very important. In our case report, IHC demonstrated that the tumor was composed of malignant epithelial component with glandular differentiation with areas of sarcoma like appearance. ${ }^{9}$ This type of tumor has a very aggressive behavior, local invasion are the striking features of this cancer. Total cystectomy is the preferred treatment modality with radiotherapy or chemotherapy, although different treatment has different consequences. ${ }^{10}$

Our case was in preliminary stage, no positive findings were in our investigations, except a cystic lesion found in front of the anterior bladder wall by USG. With this suspicion, we explored the urinary bladder with a lower midline incision and found a hard mass in the anterior bladder wall. We planned for total cystectomy but because the patient's guardian refused, so partial cystectomy was done. Post operative period was uneventful. Survival time was 7 months after surgery. Some authors found median survival time 10 months and others found 2 or 3 months after treatment. ${ }^{11,12}$ Regarding clinical outcome of sarcomatoid carcinoma of the urinary bladder very few data have been published, so it is difficult to assess due to vague diagnostic standards used in each group. Clinical behavior of such a rare tumor is difficult, but very aggressive and usually loco-regional extension is the main feature of such tumors. Total cysyectomy is the preferred method of treatment of SC of urinary bladder. ${ }^{13}$ For achievement of better local control, adjuvant radiotherapy and chemotherapy may show prospective result. ${ }^{14,15}$ Froehner et al reported a case of a metastatic SC remission of the bladder with cisplatin and gemcitabine, although in the literature, adjuvant radiotherapy or adjuvant chemotherapy are apparently of no effect. ${ }^{2,16}$ Our patient refused chemotherapy and radiotherapy, but the patient survived for 7 months after partial cystectomy due to early diagnosis and early treatment.

Conclusion: Routine investigations may not give any clue for early diagnosis of sarcomatoid carcinoma of the bladder. Total cystectomy is the choice of treatment for SC but postoperative quality of life should be considered before any aggressive decision is taken. Further study with more cases and experience may provide great value, clinically and pathologically. More study with more cases is necessary to see the survival time.

References:

1. Dahm P, Gschwend JE. Malignant non-urothelial neoplasm of the urinary bladder: a review. Eur Urol. 2003;44:672-81.

2. Luis FA, Dercilio AF, Emilio MP, Flavio LH. Sarcomatoid carcinoma with osseous differentiation in the bladder. International Braz J Urol. 2006;32(5)

3. Picazo ML, Regojo RM, Gonzalez-Peramato P. Histological variants of urothelial carcinoma with diagnostic, prognostic and therapeutic implications. Actas Urol SSP. 2007;31(9):989-1001.

4. Lopez-Beltran A, Pacelli A, Rothenberg HJ. Carcinosarcoma and sarcomatoid carcinoma of the bladder; clinicopathological study of 41 cases. J Urol. 1998;159:1497-503.

5. Knappen B ST, Uson AC, Melicon MM. Primary neoplasm occurring in vesicle diverticula: a report of 18 cases. Urol. 1960;83:153-159.

6. Sousa EA, Garcia R, Arguelles M, Maceires F, Perez-Valcarcel I. Carcinosarcoma in a bladder diverticulum: a case report and literature review. Urol Int. 2000;65:169-172.

7. Toren beek R, Blomjous CE, de Bruin PC, Newling DW, Meijer CJ. Sarcomatoid carcinoma of the urinary bladder. Clinicopathologic analysis of 18 cases with immunohistochemical and electron microscopic findings. Am J Surg Pathol. 1994; 18:241-9.

8. Ikegami H, Iwasaki H, Ohjimi Y, Takeuchi Y, Ariyoshi A, Kikuchi M. Sarcomatoid carcinoma of the urinary bladder: a clinicopathologic and immunohistochemical analysis of 14 patients. Hum Pathol. 2000;31:332-40.

9. Onal C, Pehlivan B, Bal N, Topkan E, Kilinc F, Popuk S. Sarcomatoid carcinoma of the urinary bladder treated with adjuvant radiotherapy: a case report. Clinical Medicine. 2009;2:39-42.

10. Young RH, Wick MR, Mills SE. Sarcomatoid carcinoma of the urinary bladder. A Clin Pathol. 1988;90(6):653-61.

11. Omeroglu A, Paner GP, Wojcik E M, Kalliopi S. A carcinosarcoma / sarcomatoid carcinoma arising in a urinary bladder diverticulam. Arch Pathol Lab Med. 2002;126:853-55.

12. Eble JN, Young RH. Carcinoma of the urinary bladder: a review of its diverse morphology. Semin Diagn Pathol. 1997;14:98-108.

13. Chen KT. Carcinosarcoma of the bladder. Arch Pathol Lab Med. 1992;116:811.

14. Ogishima T, Kawachi Y, Saito A, Suzuki F, Ishi K, Tanaka T. Sarcomatoid carcinoma and carcinosarcoma of the urinary bladder. Int J Urol. 2002;9:354-8.

15. Yeniyol CO, Tuna A, Yagci A, Ozguder O, Postachi H. Human Chorionic gonadotropin in sarcomatoid carcinoma of the bladder. A case report. Int Urol Nephrol. 2003;35:29-31.

16. Froehner M, Gaertner HJ, Manseck A, Wirth MP. Durable complete remission of metastatic sarcomatoid carcinoma of the bladder with cisplatin and gemcitabine in an 80-year-old man. Urology. 2001;58:799.

Fig.1 High power view of Sarcomatoid carcinoma. It is composed of malignant epithelial component with glandular differentiation and area having sarcoma like appearance. ( H and E x 200) 